\title{
The leptonic CP phase from muon decay at rest with two detectors
}

\author{
Emilio Ciuffoli, ${ }^{a, b}$ Jarah Evslin ${ }^{a, b}$ and Xinmin Zhang ${ }^{a, c}$ \\ a TPCSF, IHEP, CAS, \\ Beijing 100049, China \\ ${ }^{b}$ Institute of Modern Physics, CAS, \\ NanChangLu 509, Lanzhou 730000, China \\ ${ }^{c}$ Theoretical physics division, IHEP, CAS, \\ Beijing 100049, China \\ E-mail: ciuffoli@ihep.ac.cn, jarah@ihep.ac.cn, xmzhang@ihep.ac.cn
}

ABSTRACT: We propose a novel experimental setup for the determination of the leptonic CP-violating phase $\delta$ using the decay at rest (DAR) of $\mu^{+}$from a single source located at distances of 10 and $30 \mathrm{~km}$ from two $20 \mathrm{kton}$ organic liquid scintillator detectors. The $\mu^{+}$are created by bombarding a target with a $9 \mathrm{~mA}$ beam of $800 \mathrm{MeV}$ protons. With this proposal $\delta$ can be determined with a precision of 20 (15) degrees in 6 (12) years. In contrast with the DAE $\delta$ ALUS project, only a single source is required and it runs with a duty factor limited only by maintenance requirements. As a result $9 \mathrm{~mA}$ is the maximum instantaneous current, greatly reducing both the technological challenges and the costs.

Keywords: Neutrino Detectors and Telescopes, CP violation

ARXIV EPRINT: 1401.3977 
The recent discovery that the last neutrino mass mixing angle $\theta_{13}$ is nonzero implies that leptonic interactions may break CP symmetry. In particular, neutrino oscillations are sensitive to a phase $\delta$ in the mass matrix which yields a $\mathrm{CP}$ violation proportional to $\sin (\delta)$. Today the value of $\delta$ is entirely unknown. In the next decade the experiments $\mathrm{NO} \nu \mathrm{A}$ and $\mathrm{T} 2 \mathrm{~K}$ will have some sensitivity to $\sin (\delta)$ but they will not be able to distinguish $\delta$ from $\pi-\delta[1,2]$. Future proposals in general are expensive and depend on unproven technology, such as the scalability of liquid argon detectors and the control of excitations of $\mathrm{H}_{2}^{+}$. Below we describe a proposal which will yield a 20 (15) degree precision measurement of $\delta$ in 6 (12) years using technology not far beyond the state of the art and at a much lower cost than its competitors.

A cyclotron complex, consisting of pairs of cyclotrons, will accelerate protons to 800 $\mathrm{MeV}$ which then strike a target, producing $\pi^{+}$that decay at rest (DAR). The resulting $\mu^{+}$ will in turn decay at rest creating $\bar{\nu}_{\mu}$ that then oscillate to $\bar{\nu}_{e}$. These $\bar{\nu}_{e}$ are detected via inverse $\beta$ decay (IBD) by two organic liquid scintillator detectors, each with a target mass of $20 \mathrm{kton}$ and consisting of $12 \%$ free protons, located 10 and $30 \mathrm{~km}$ from the complex.

Existing and proposed accelerator neutrino experiments, such as T2K, T2HK, $\mathrm{NO} \nu \mathrm{A}$, LBNE(F), LBNO and Daya Bay 3 attempt to measure $\delta$ using $\nu_{e}$ appearance in a $\nu_{\mu}$ beam at the first oscillation maximum. At the first oscillation maximum, the transition probability from $\mu$ to $e$ (anti)neutrinos is

$$
P=\sin ^{2}\left(2 \theta_{13}\right) \sin ^{2}\left(\theta_{23}\right) \mp \frac{\pi}{2} \frac{\Delta M_{21}^{2}}{\left|\Delta M_{31}^{2}\right|} \sin (2 \theta 12) \sin (2 \theta 13) \sin (2 \theta 23) \cos \left(\theta_{13}\right) \sin (\delta)
$$

where the $-(+)$ sign is for (anti)neutrinos. Including the best fit values of these parameters but excluding T2K, which will be treated extensively below, this is

$$
P_{\nu_{\mu} \rightarrow \nu_{e}} \sim(0.045 \pm 0.010)-0.014 \sin (\delta), \quad P_{\bar{\nu}_{\mu} \rightarrow \bar{\nu}_{e}} \sim(0.045 \pm 0.010)+0.014 \sin (\delta) .
$$

Notice that the uncertainty on the first term, $\sin ^{2}\left(2 \theta_{13}\right) \sin ^{2}\left(\theta_{23}\right)$, is not much smaller than the value of the entire second term, which provides the $\delta$ dependence.

This means that, with current uncertainties, one cannot measure $\delta$ using only neutrino or antineutrino oscillations at the first maximum, as there is a degeneracy between $\sin ^{2}\left(2 \theta_{13}\right) \sin ^{2}\left(\theta_{23}\right)$ and $\sin (\delta)$. One way to evade this problem, which $\mathrm{NO} \nu \mathrm{A}$ and some other experiments will persue, is to run sometimes in $\nu$ mode and sometimes in $\bar{\nu}$ mode. Combining the results with eq. (1) one can in principle determine $\sin ^{2}\left(2 \theta_{13}\right) \sin ^{2}\left(\theta_{23}\right)$ and $\sin (\delta)$ separately. The disadvantage with this approach is that, since the accelerators use proton beams, they create more $\pi^{+}$than $\pi^{-}$, whose decay creates $\nu_{\mu}$ and not $\bar{\nu}_{\mu}$. Therefore, the luminosity per proton on target is much higher in the $\nu$ mode than in the $\bar{\nu}$ mode. This low luminosity in the $\bar{\nu}$ mode then leads to a large statistical uncertainty in these experiments. In particular a $3 \sigma$ discovery of $\mathrm{CP}$ violation at $\mathrm{NO} \nu \mathrm{A}$ seems challenging. Furthermore, the oscillation probabilty at the oscillation maximum, given in eq. (1) depends only upon $\sin (\delta)$ and so cannot distinguish $\delta$ from $\pi-\delta$. 
A $\mu^{+}$DAR experiment can address all of these problems. First of all, unlike an offaxis beam experiment, the $\mu$ DAR neutrino spectrum is not monochromatic and so the measurement is not restricted to the oscillation maximum. Therefore, as we will see below, $\delta$ and $\pi-\delta$ can be cleanly distinguished. Next, $\mu^{+}$DAR operates entirely in the $\bar{\nu}$ mode. As a result, on its own, such an experiment may struggle with the same degeneracy between $\sin ^{2}\left(2 \theta_{13}\right) \sin ^{2}\left(\theta_{23}\right)$ and $\sin (\delta)$ as an accelerator experiment running in $\nu$ mode. However, as can be seen in eq. (1), in the $\bar{\nu}$ mode this degeneracy has an opposite relative sign and so, when combined with the data which will be provided by $\mathrm{T} 2 \mathrm{~K}$ and $\mathrm{NO} \nu \mathrm{A}$ in $\nu$ mode during the next 5 years, the degeneracy will be broken: $\sin ^{2}\left(2 \theta_{13}\right) \sin ^{2}\left(\theta_{23}\right)$ and $\sin (\delta)$ will each be determined precisely, the first roughly from the sum of $\nu_{e}$ and $\bar{\nu}_{e}$ appearance at accelerator and $\mu^{+}$DAR experiments and the second from the difference. Below we will quantify the resulting precision. As $\mathrm{T} 2 \mathrm{~K}$ and $\mathrm{NO} \nu \mathrm{A}$ both have a higher luminosity in $\nu$ mode, optimally, if a $\mu$ DAR experiment is planned, the precision will be optimized if they use all of their beam time in the $\nu$ mode. In this sense, a $\mu$ DAR experiment has a maximal synergy with an accelerator $\nu$ experiment and when both are combined the determination of $\delta$ will be quite precise.

Which baselines are optimal for a $\mu$ DAR experiment? Recall that we have proposed one $\mu \mathrm{DAR}$ source and two detectors, so there are two baselines to fix. We have simulated all combinations of these baselines. Although the optimal baseline is somewhat dependent upon $\delta$, in general we have found that the best precision in a measurement of $\delta$ is obtained with baselines of roughly $10 \mathrm{~km}$ and $30 \mathrm{~km}$. This result is fairly insenstive to the magnitude of the atmospheric $\nu_{e}$ background that is expected at a scintillator detector in southeast or east Asia. Why these baselines? At a $30 \mathrm{~km}$ baseline the $\mu$ DAR spectrum covers the area around the oscillation maximum, providing an excellent sensitivity to $\sin (\delta)$. The detector at $10 \mathrm{~km}$ from the source detects the neutrinos well before they have reached the first oscillation peak, and so is sensitive to a quite distinct and complimentary superposition of $\sin (\delta)$ and $\cos (\delta)$ as will be seen in figure 1. Combining these two measurements, both $\sin (\delta)$ and $\cos (\delta)$ can be determined individually, yielding the phase $\delta$ with no remaining degeneracies.

We will see below that the precision with which our proposal can measure $\delta$ is competitive with other proposals. Our proposal requires a much less expensive accelerator than most competing proposals, but does require two detectors. Is it therefore more expensive? The primary motivation for our proposal is that such pairs of detectors, with just the separation that we need, may anyway be built. As has been proposed in refs. [4-6], such a pair of detectors may be employed by the JUNO [7] and RENO 50 [8] experiments to determine the neutrino mass hierarchy using reactor neutrinos. The employment of a pair of detectors eliminates the loss of sensitivity to the mass hierarchy resulting from the detector's unknown nonlinear energy response.

The principle behind the DAE $\delta$ ALUS project is similar but its manifestations in refs. $[3$, $9,10]$ each require three cyclotron complexes, each consisting of one or more modules [11] which each in turn consist of a cyclotron pair. At these energies it is difficult to determine which neutrino arrived from which complex, therefore only one complex may run at a time. This limitation is problematic as these proposals are always statistics limited, even when the detector is as large as Hyper-K [10]. Thus our proposal, with a single $\mu^{+}$source, enjoys the same neutrino flux as would a proposal with three identical sources. As in the case 

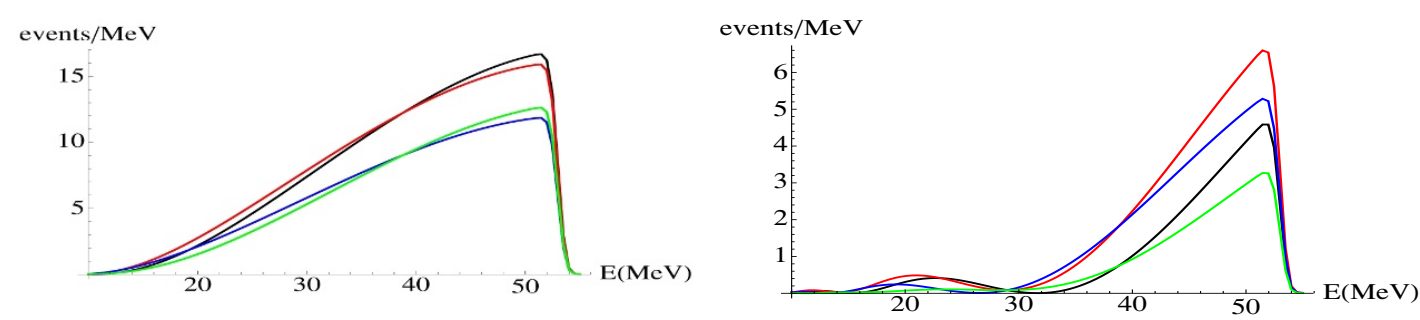

Figure 1. Number of $\bar{\nu}_{e}$ per $\mathrm{MeV}$ expected at the near detector (left) and the far detector (right) in 6 years if $\delta=0^{\circ}$ (black), $90^{\circ}$ (red), $180^{\circ}$ (blue) and $270^{\circ}$ (green).

of DAE $\delta$ ALUS [11], the physics goals are not compromised if the total current is divided among multiple modules separated by less than 100 meters, each with its own target.

The determination of $\delta$ using $\mu^{+}$DAR has several advantages. For one, the neutrino spectrum and IBD cross section are known precisely. Second, in the window between $20 \mathrm{MeV}$ and $55 \mathrm{MeV}$ the $\bar{\nu}_{e}$ backgrounds are small. The main background arises from atmospheric $\nu_{e}$ and $\bar{\nu}_{e}$. For example, $20-55 \mathrm{MeV} \bar{\nu}_{e}$ may interact with free protons in the scintillator via IBD yielding an irreducible background which, event for event, is indistinguishable from our signal. Similarly $\bar{\nu}_{e}$ with energies of order $100 \mathrm{MeV}$ may undergo quasielastic interactions with protons bound in ${ }^{12} \mathrm{C}$ in the scintillator and in general such interactions liberate a neutron and positron and so yield a double coincidence. As a result of the binding energy of the proton, the resulting positron often has an energy in the signal range and so again this results in an irreducible background. Finally, $\nu_{e}$ with energies of order $100 \mathrm{MeV}$ which interact quasielastically with neutrons in the ${ }^{12} \mathrm{C}$ in general do not liberate a neutron but nonetheless sometimes do. As atmospheric $\nu_{e}$ outnumber atmospheric $\bar{\nu}_{e}$ and enjoy a higher quasielastic cross-section, the emitted neutron and electron also create a nonnegligible background. While these backgrounds are irreducible, they are reasonably small and they have a reasonably flat energy spectrum which extends beyond the DAR decay spectrum. Using the fact that the signal DAR spectrum is known, a shape analysis can be used to effectively remove these backgrounds [12]. Unlike DAE $\delta$ ALUS at Hyper-K, as the detectors are liquid scintillators, there is no invisible muon background, on the contrary the JUNO detector is sensitive to muons at very low energies.

Third, as this energy range is distinct from the $2-8 \mathrm{MeV}$ energy range of the reactor neutrinos used to determine the mass hierarchy, the reactor and $\mu^{+}$DAR experiments may run simultaneously. In fact the mass hierarchy experiment will start several years earlier, allowing a reasonable understanding of the backgrounds. Finally, this determination uses $\bar{\nu}$ oscillations, which have maximum synergy with accelerator experiments like T2K, $\mathrm{NO} \nu \mathrm{A}$ and LBNE that enjoy better statistics in the $\nu$ oscillation channel. By comparing the two channels one can remove the degeneracy between $\delta$, which can be extracted from the difference between the appearance in the neutrino and antineutrino channels, and $\sin ^{2}\left(2 \theta_{13}\right) \sin ^{2}\left(\theta_{23}\right)$, which increases the appearance in both channels simultaneously and so can be extracted from the sum of the appearance rates.

In the calculations below we have fixed the normalization of the number of IBD events such that, with $\delta=0$, at $10 \mathrm{~km}$ a 20 kton target mass detector, consisting of $12 \%$ free 
protons, will observe 350 events in 6 years. Scaling the $\mu^{+}$decay rate at LSND [13], this corresponds to about $45 \mathrm{MW}$ years of power using a $800 \mathrm{MeV}$ proton beam, or equivalently $1.8 \times 10^{6} \mathrm{C}$ of protons. For example, for a 6 year run one would require a constant current of $9 \mathrm{~mA}$. For simplicity below we will assume a constant current of $9 \mathrm{~mA}$ and report the livetime.

The critical advantage of our proposal is that the duty factor of the cyclotron is limited only by maintenance requirements, so $9 \mathrm{~mA}$ is not only the average current, but also the peak instaneous current. The DAE $\delta$ ALUS far complex alone will have an average power of $5 \mathrm{MW}$ over 10 years [3] which, with a $20 \%$ duty factor, implies that the product of the peak power and livetime at that complex is $250 \mathrm{MW}$ years, more than 5 times that of our entire proposal. In neither case does the number of years include the downtime required for maintenance.

As a result, the technological requirements on our cyclotrons are relatively modest. For example, two modules, each using the cyclotron proposed in ref. [15], would be more than sufficient. On the other hand target cooling depends upon the average and not the peak power and so will be challenging. One promising proposal is a conical graphite beam dump inside of a copper mantle [16].

As explained in ref. [10] a factor of 2 in the beam power at fixed perveance may be gained by accelerating $H_{2}^{+}$. This is challenging as the $H_{2}^{+}$excited states need to be controlled. As a result of our lower beam power requirements, a proton beam may well be sufficient for this proposal.

In the current study we have restricted our attention to the normal neutrino mass hierarchy, considered only the tree level IBD cross section and ignored neutron recoil. However we have included matter effects as the neutrinos travel through the Earth.

We fix the neutrino mass differences to be

$$
\Delta M_{31}^{2}=2.4 \times 10^{-3} \mathrm{eV}^{2}, \quad \Delta M_{21}^{2}=7.5 \times 10^{-5} \mathrm{eV}^{2}
$$

and the neutrino mixing angles to be

$$
\sin ^{2}\left(2 \theta_{13}\right)=0.089, \sin ^{2}\left(2 \theta_{12}\right)=0.857, \sin ^{2}\left(\theta_{23}\right)=\frac{1}{2} .
$$

The effect of the uncertainty in the mixing angles will be studied below while in ref. [12] it will be shown that the uncertainty in the mass splittings, which will be greatly reduced by JUNO, will be have a small effect on the determination of $\delta$.

The expected spectra at the near $(10 \mathrm{~km})$ and far $(30 \mathrm{~km})$ detectors after a 6 year run are plotted in figure 1 for $\delta=0^{\circ}, 90^{\circ}, 180^{\circ}$ and $270^{\circ}$. Note that the differences in shape of the spectra are fairly small, but that the energy resolution of a liquid scintillator detector is quite good in this energy range. In fact JUNO and RENO 50, in part because of the density and quality of their PMTs, are expected to have an energy resolution as much as a factor of two better than LENA. We used a somewhat conservative fractional energy resolution

$$
\frac{\delta E}{E}=\sqrt{\left(\frac{3 \%}{\sqrt{E / \mathrm{MeV}}}\right)^{2}+(1 \%)^{2}} .
$$



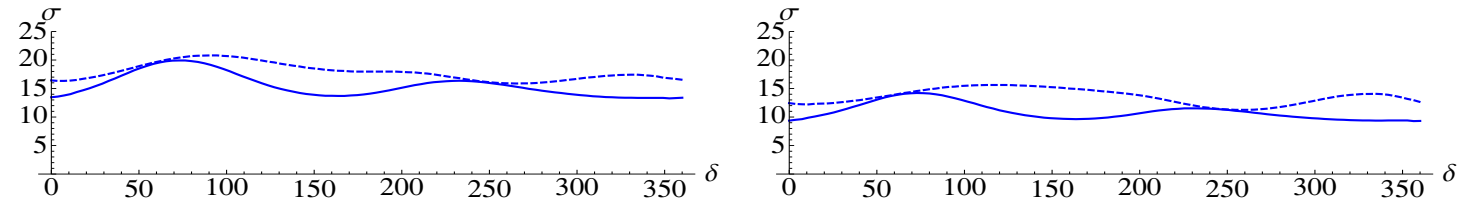

Figure 2. The $1 \sigma$ precision, in degrees, with which $\delta$ can be determined for various values of $\delta$ in a 6 year (left) and 12 year (right) run. In this figure we have assumed that the mass matrix mixing angles are known perfectly. The total normalization of the $\bar{\nu}_{\mu}$ flux is known perfectly (solid curve) and with a precision of $5 \%$ (dashed).

We determine the $1 \sigma$ precision with which these experiments may determine $\delta$ using a Poisson-statistics $\chi^{2}$ fit to the Asimov data set. In figure 2 we report this precision for 6 and 12 years of running. In this figure we assume that the mixing angles are known perfectly. To test these results we have also performed a series of Monte Carlo simulations for each mixing angle. The results of our Monte Carlo are compatible with those of the $\chi^{2}$ analysis.

We have considered various uncertainties in the overall normalization of the neutrino flux, treated using the standard pull parameter method of ref. [17]. A 20\% uncertainty may be expected based simply on dead reckoning. A 5\% uncertainty may be obtained using, for example, neutrino electron scattering at a near detector. For example one of the eight 20 ton liquid scintillator detectors used at Daya Bay may be placed 50-100 meters from the complex equidistant from each module. Such a near detector would also be sensitive to sterile neutrinos in a parameter space far exceeding that of LSND.

In figure 3 we include uncertainties in the mixing angles corresponding to the current uncertainties

$$
\begin{aligned}
\delta \sin ^{2}\left(2 \theta_{12}\right) & =0.024, \quad \delta \sin ^{2}\left(2 \theta_{13}\right)=0.01 \\
\frac{\delta \sin \left(\theta_{23}\right)}{\sin \left(\theta_{23}\right)} & =11 \%
\end{aligned}
$$

and also with uncertainties expected when experiments currently running are finished

$$
\begin{aligned}
\frac{\delta \sin ^{2}\left(2 \theta_{12}\right)}{\sin ^{2}\left(2 \theta_{12}\right)} & =1 \%, \quad \frac{\delta \sin ^{2}\left(2 \theta_{13}\right)}{\sin ^{2}\left(2 \theta_{13}\right)}=4 \% \\
\delta \sin \left(\theta_{23}\right) & =0.02 .
\end{aligned}
$$

To understand the relevant contributions of the uncertainties from the different mixing angles, in figure 4 we have fixed all of the angles except for one, to which we have applied the current and future uncertainties. The main contribution to the uncertainty arises from a single combination, $\sin ^{2}\left(2 \theta_{13}\right) \sin ^{2}\left(\theta_{23}\right)$, of $\theta_{13}$ and $\theta_{23}$. This degeneracy will be broken by combining data from $\mu^{+}$DAR with the $\nu_{e}$ appearance channel at accelerator experiments.

Our main result is figure 3. As can be seen, a single cyclotron complex, producing antineutrinos using $\mu^{+}$DAR, can determine $\delta$ with a precision of 20 (15) degrees in 6 (12) years using detectors that may anyway be built for reactor neutrino experiments. 

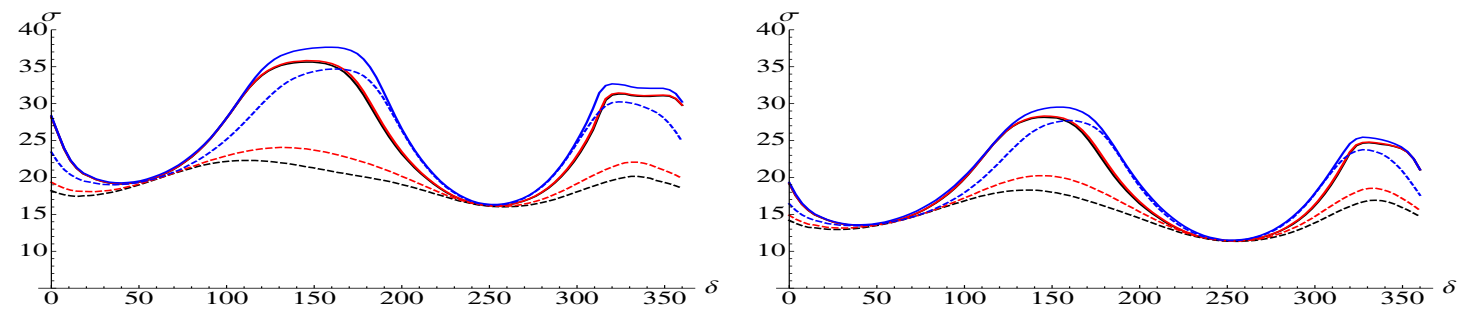

Figure 3. The $1 \sigma$ precision, in degrees, with which $\delta$ can be determined for various values of $\delta$ in a 6 year (left) and 12 year (right) run. The total normalization of the $\bar{\nu}_{\mu}$ flux is known with a precision of $1 \%$ (black), $5 \%$ (red) and $20 \%$ (blue). The solid (dashed) curves correspond to current (future) uncertainties in the mixing angles.
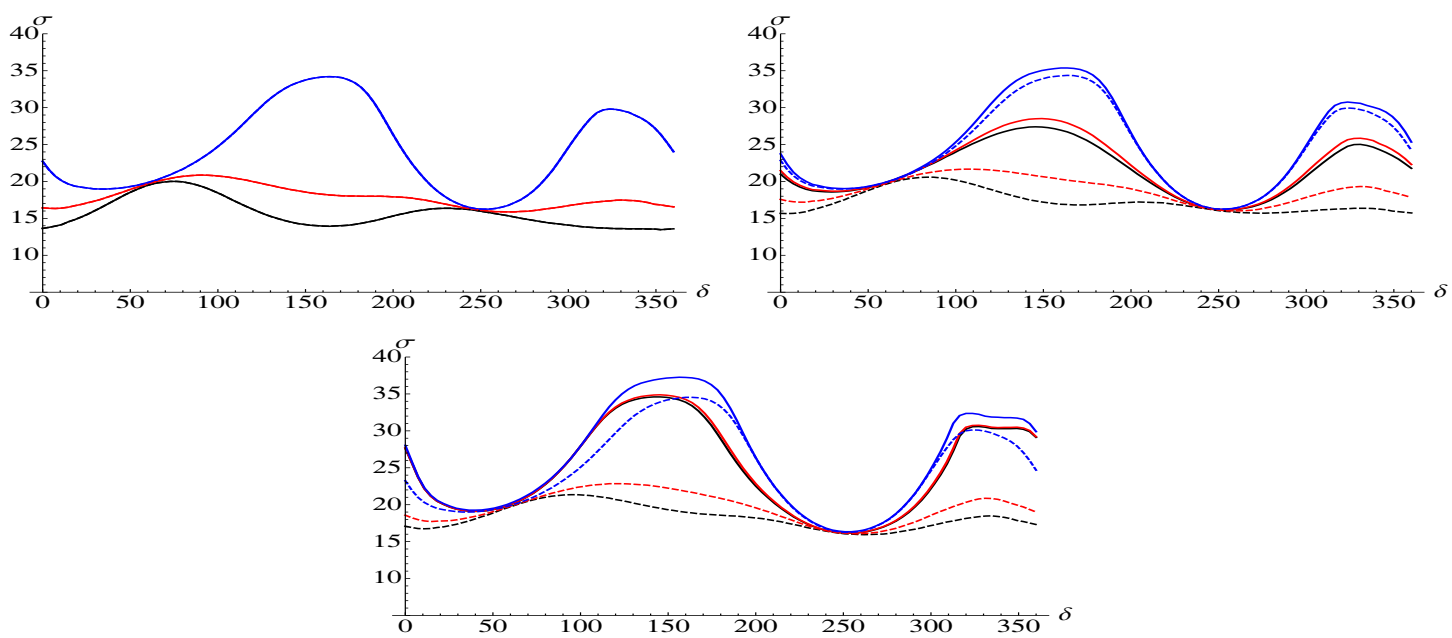

Figure 4. Six years of running, as in figure 3 except that all of the mixing angles are fixed except for $\theta_{12}$ (top left), $\theta_{13}$ (top right) and $\theta_{23}$ (bottom). Note that the error in $\theta_{12}$ has essentially no effect on the precision with which $\delta$ can be determined.

The main background arises from atmospheric $\bar{\nu}_{e}$ which interact via IBD with $\mathrm{H}$ in the scintillator or quasielastically with ${ }^{12} \mathrm{C}$ and from the far more common atmospheric $\nu_{e}$ interactions with ${ }^{12} \mathrm{C}$ which occasionally liberate a neutron, yielding a false double coincidence. The strong horizontal magnetic field over southern China implies that these backgrounds will be reduced by almost a factor of two with respect to Homestake and Pyhäsalmi mines [18, 19]. In ref. [12] we will show that in 6 years, $9 \bar{\nu}_{e}$ and $2 \nu_{e}$ background events yield prompt energies in the signal range of $40-55 \mathrm{MeV}$. This is smaller than the expected signal and can be reduced with a shape analysis and also using the rate measured at JUNO before the cyclotrons are built and also above the high energy cutoff of the $\mu$ DAR spectrum. $\gamma$ emitted by de-exciting ${ }^{12} \mathrm{C}$ can also be used to veto the quasielastic background events [12]. Therefore in this study we have not included the background.

In refs. [1, 2] it was noted that off axis accelerator experiments designed to measure $\delta$ have relatively monochromatic beams and so are sensitive primarily to the flux at the oscillation maximum, which depends only upon $\sin (\delta)$ and so cannot distinguish $\delta$ from $180^{\circ}-\delta$. On the other hand, the $\mu^{+}$DAR spectrum is far from monochromatic and so there is no such degeneracy in the $\delta$ determined by such experiments. This can be seen in 

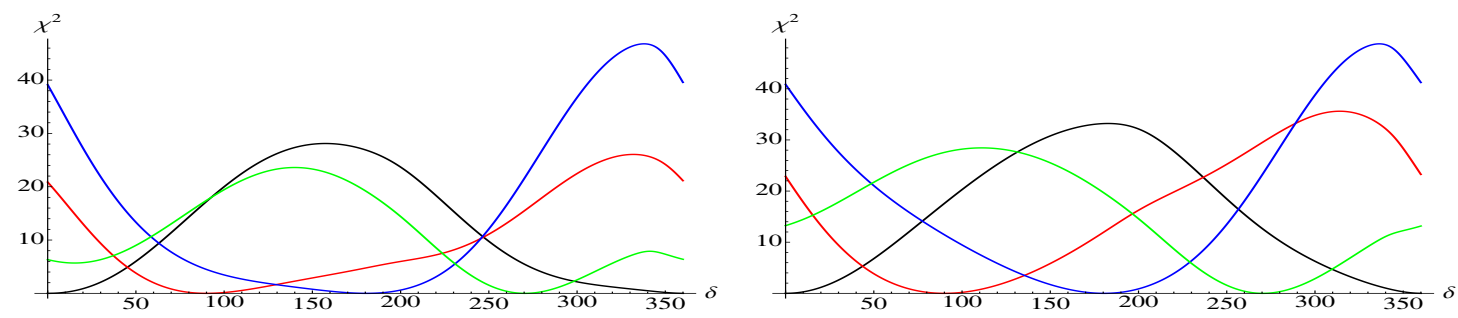

Figure 5. The true values of $\delta$ are $0^{\circ}$ (black), $90^{\circ}$ (red), $180^{\circ}$ (blue) and $270^{\circ}$ (green). The reported values of $\chi^{2}$ correspond to fits of 6 years of data to various values of $\delta$ assuming a $5 \%$ uncertainty in the normalization and the current (left panel) and future (right panel) uncertainties in the mixing angles. Note that there is essentially no degeneracy between $\delta$ and $180^{\circ}-\delta$.

figure 5 where $\chi^{2}$ is plotted as a function of the $\delta$ in the fitting function, the degeneracy would correspond to a local minimum at the $180^{\circ}$ minus the true value of $\delta$. No such minimum is present in the figures.

\section{Acknowledgments}

We have benefited from discussions with Jianjun Yang and Tianjue Zhang. We thank ShaoFeng Ge for finding an error in an earlier version of this manuscript. JE is supported by NSFC grant 11375201. EC is supported by the CAS grant 2013Y1JB0001 and NSFC grant 11350110500. XZ is supported in part by NSFC grants 11121092, 11033005 and 11375202.

Open Access. This article is distributed under the terms of the Creative Commons Attribution License (CC-BY 4.0), which permits any use, distribution and reproduction in any medium, provided the original author(s) and source are credited.

\section{References}

[1] E. Ciuffoli, J. Evslin and X. Zhang, Neutrino mass hierarchy from nuclear reactor experiments, Phys. Rev. D 88 (2013) 033017 [arXiv:1302.0624] [InSPIRE].

[2] H. Minakata and S.J. Parke, Correlated, precision measurements of $\theta_{23}$ and $\delta$ using only the electron neutrino appearance experiments, Phys. Rev. D 87 (2013) 113005 [arXiv: 1303.6178] [INSPIRE].

[3] J. Alonso et al., Expression of interest for a novel search for CP-violation in the neutrino sector: DAESALUS, arXiv: 1006.0260 [INSPIRE].

[4] E. Ciuffoli, J. Evslin and X. Zhang, Mass hierarchy determination using neutrinos from multiple reactors, JHEP 12 (2012) 004 [arXiv:1209.2227] [INSPIRE].

[5] E. Ciuffoli et al., Medium baseline reactor neutrino experiments with two identical detectors, Phys. Lett. B 736 (2014) 110 [arXiv:1211.6818] [INSPIRE].

[6] E. Ciuffoli et al., Advantages of multiple detectors for the neutrino mass hierarchy determination at reactor experiments, Phys. Rev. D 89 (2014) 073006 [arXiv:1308.0591] [INSPIRE]. 
[7] Y.-F. Li, J. Cao, Y. Wang and L. Zhan, Unambiguous determination of the neutrino mass hierarchy using reactor neutrinos, Phys. Rev. D 88 (2013) 013008 [arXiv:1303.6733] [INSPIRE].

[8] S.B. Jim, Proposal for RENO-50; detector design 8 goals, talk given at the International Workshop on RENO-50: Towards the Neutrino Mass Hierarchy, June 13-14, Seoul National University, Seoul, Korea (2013).

[9] LENA collaboration, M. Wurm et al., The next-generation liquid-scintillator neutrino observatory LENA, Astropart. Phys. 35 (2012) 685 [arXiv:1104.5620] [INSPIRE].

[10] C. Aberle et al., Whitepaper on the DAESALUS program, arXiv:1307.2949 [INSPIRE].

[11] M. Absw et al., Multimegawatt DAE $\delta A L U S$ cyclotrons for neutrino physics, arXiv: 1207.4895 [INSPIRE].

[12] J. Evslin, S.F. Ge and K. Hagiwara, The leptonic CP phase from T2(H)K and muon decay at rest, work in progress.

[13] LSND collaboration, A. Aguilar-Arevalo et al., Evidence for neutrino oscillations from the observation of anti-neutrino(electron) appearance in a anti-neutrino(muon) beam, Phys. Rev. D 64 (2001) 112007 [hep-ex/0104049] [INSPIRE].

[14] J. Alonso, High current H2+ cyclotrons for neutrino physics: the IsoDAR and DAESALUS projects, AIP Conf. Proc. 1525 (2013) 480 [arXiv:1210.3679] [INSPIRE].

[15] T. Zhang et al., Conceptual design of an $800 \mathrm{MeV}$ high power proton driver, Nucl. Instrum. Meth. B 269 (2011) 2964.

[16] C. Tschalär, High-power proton beam dump with uniform graphite depth, Bates Preprint B/IR\#12-01 (2012).

[17] D. Stump, J. Pumplin, R. Brock, D. Casey, J. Huston et al., Uncertainties of predictions from parton distribution functions. 1. The lagrange multiplier method, Phys. Rev. D 65 (2001) 014012 [hep-ph/0101051] [INSPIRE].

[18] G. Barr, T.K. Gaisser and T. Stanev, Flux of atmospheric neutrinos, Phys. Rev. D 39 (1989) 3532 [INSPIRE].

[19] M. Sajjad Athar, M. Honda, T. Kajita, K. Kasahara and S. Midorikawa, Atmospheric neutrino flux at INO, South Pole and Pyhasalmi, Phys. Lett. B 718 (2013) 1375 [arXiv: 1210.5154] [INSPIRE]. 